\title{
PRESHOWER
}

\section{LEAD THICKNESS MEASUREMENTS}

D-ZERO ENGINEERING NOTE \# 3823.114-EN-486

February 16, 1998

Author: Russ Rucinski

PPD/ETT/D-Zero Upgrade project 
The preshower lead thickness applied to the outside of D-Zero's superconducting solenoid vacuum shell was measured at the time of application. This engineering documents those thickness measurements.

The lead was ordered in sheets 0.09375 " and 0.0625 " thick. The tolerance on thickness was specified to be $+/-0.003 "$. The sheets all were within that thickness tolerance.

The nomenclature for each sheet was designated $1 \mathrm{~T}, 1 \mathrm{~B}, 2 \mathrm{~T}, 2 \mathrm{~B} \ldots$ where the numeral designates it's location in the wrap and " $T$ " or " $B$ " is short for 'top' or 'bottom' half of the solenoid.

Micrometer measurements were taken at six locations around the perimeter of each sheet. The width, length, and weight of each piece was then measured. Using an assumed pure lead density of $0.40974 \mathrm{lb} / \mathrm{in}^{\wedge} 3$, an average sheet thickness was calculated and compared to the perimeter thickness measurements.

In every case, the calculated average thickness was a few mils thinner than the perimeter measurements. The ratio was constant, 0.98 . This discrepancy is likely due to the assumed pure lead density. It is not felt that the perimeter is thicker than the center regions. The data suggests that the physical thickness of the sheets is uniform to $+/$ $0.0015 "$. 
PIECE DESKNATION

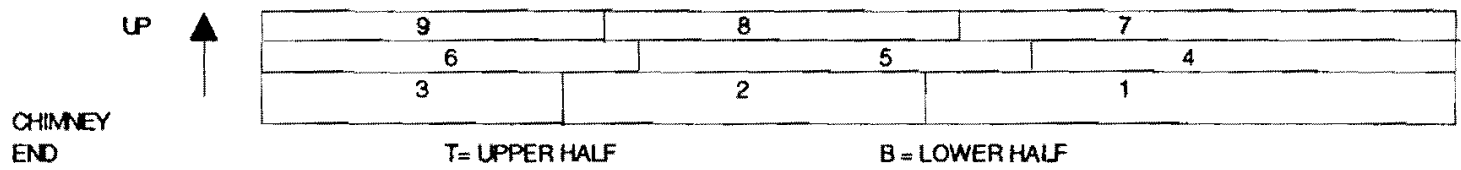

Specification on thickness $=+1-0.003$

\begin{tabular}{|c|c|c|c|c|c|c|c|c|}
\hline PECEE & NOM. DIMENSIONS & $\begin{array}{l}\text { MEASURED } \\
\text { LENGTH } \\
\text { (In.) }\end{array}$ & $\begin{array}{l}\text { MEASURED } \\
\text { WIDTH } \\
\text { (1n.) }\end{array}$ & $\begin{array}{c}\text { MEASURED } \\
\text { WT. OF } \\
\text { LEAD+CARRIER }\end{array}$ & $\begin{array}{l}\text { MEASURED } \\
\text { WT. OF } \\
\text { CARPEA }\end{array}$ & $\begin{array}{l}\text { Net LEAD } \\
W T .(1 b s)\end{array}$ & $\begin{array}{l}\text { AVG. THKNS } \\
\text { MEASURED } \\
\text { AT EDGE }\end{array}$ & $\begin{array}{l}\text { CALC. AVG. } \\
\text { THICKNESS } \\
\text { (BY WT.) }\end{array}$ \\
\hline $1 T$ & $3 / 32 \times 36 \times 873 / 16$ & 87.375 & 36.0625 & 158.7 & 42.8 & 115.9 & 0.0913 & 0.0897 \\
\hline $1 B$ & $3 / 32 \times 36 \times 873 / 16$ & 87.375 & 36.0625 & 161.1 & 42.8 & 118.3 & 0.0938 & 0.0916 \\
\hline $2 T$ & $3 / 32 \times 38 \times 873 / 16$ & 87.375 & 36.0625 & 160.3 & 42.8 & 117.5 & 0.0932 & 0.0909 \\
\hline 2B & $3 / 32 \times 36 \times 873 / 16$ & 87.375 & 36.0625 & 160.2 & 42.8 & 117.4 & 0.0925 & 0.0909 \\
\hline $3 T$ & $3 / 32 \times 24 \times 873 / 16$ & 87.375 & 24.03125 & 123.1 & 42.8 & 80.3 & 0.0943 & 0.0933 \\
\hline $3 \mathrm{~B}$ & $3 / 32 \times 24 \times 873 / 16$ & 87.25 & 24.0625 & 120.6 & 42.8 & 77.8 & 0.0918 & 0.0904 \\
\hline $4 T$ & $1 / 16 \times 24 \times 883 / 16$ & 88.375 & 24.125 & 97.9 & 42.8 & 55.1 & 0.0635 & 0.0630 \\
\hline $4 B$ & $1 / 16 \times 24 \times 883 / 16$ & 88.375 & 24.125 & 98 & 42.8 & 55.2 & 0.064 & 0.0631 \\
\hline $5 T$ & $1 / 16 \times 36 \times 883 / 16$ & 88.375 & 36 & 121.8 & 42.8 & 79 & 0.0615 & 0.0606 \\
\hline $5 \mathrm{~B}$ & $1 / 16 \times 36 \times 883 / 16$ & 88.3125 & 36 & 124.3 & 42.8 & 81.5 & 0.063 & 0.0625 \\
\hline $6 \mathrm{~T}$ & $1 / 16 \times 36 \times 883 / 16$ & 88.375 & 36 & 122.7 & 42.8 & 79.9 & 0.0625 & 0.0612 \\
\hline $6 \mathrm{~B}$ & $1 / 16 \times 36 \times 883 / 16$ & 88.375 & 36 & 122.2 & 42.8 & 79.4 & 0.063 & 0.0609 \\
\hline $7 T$ & $1 / 16 \times 34 \times 883 / 8$ & 88.625 & 34 & 122.6 & 42.8 & 79.8 & 0.0655 & 0.0646 \\
\hline
\end{tabular}




\begin{tabular}{|c|c|c|c|c|c|c|c|c|}
\hline 78 & $1 / 16 \times 34 \times 883 / 8$ & 88.4375 & 34 & 121.5 & 42.8 & 78.7 & 0.0653 & 0.0638 \\
\hline $8 \mathrm{~T}$ & $1 / 16 \times 34 \times 883 / 8$ & 88.625 & 34 & 120.6 & 42.7 & 77.9 & 0.0643 & 0.0631 \\
\hline 8日 & $1 / 16 \times 34 \times 883 / 8$ & 88.5625 & 34 & 117.9 & 42.7 & 75.2 & 0.06283 & 0.0609 \\
\hline $9 T$ & $1 / 16 \times 28 \times 883 / 8$ & 88.625 & 28 & 104.9 & 42.7 & 62.2 & 0.0615 & 0.0611 \\
\hline $9 B$ & $1 / 16 \times 28 \times 883 / 8$ & 88.5625 & 28 & 104.4 & 42.7 & 61.7 & 0.0615 & 0.0607 \\
\hline THICK SPARE & $9 / 32 \times 36 \times 879 / 16$ & 87.375 & 36.0625 & 158.2 & 42.8 & 115.4 & 0.0907 & 0.0893 \\
\hline THIN SPARE & $1 / 16 \times 34 \times 883 / 8$ & & & & & & & \\
\hline
\end{tabular}

MEASUREMENTS MADE BY PAT HEALY \& CRAIG ROGERS, $2 / 90$ 


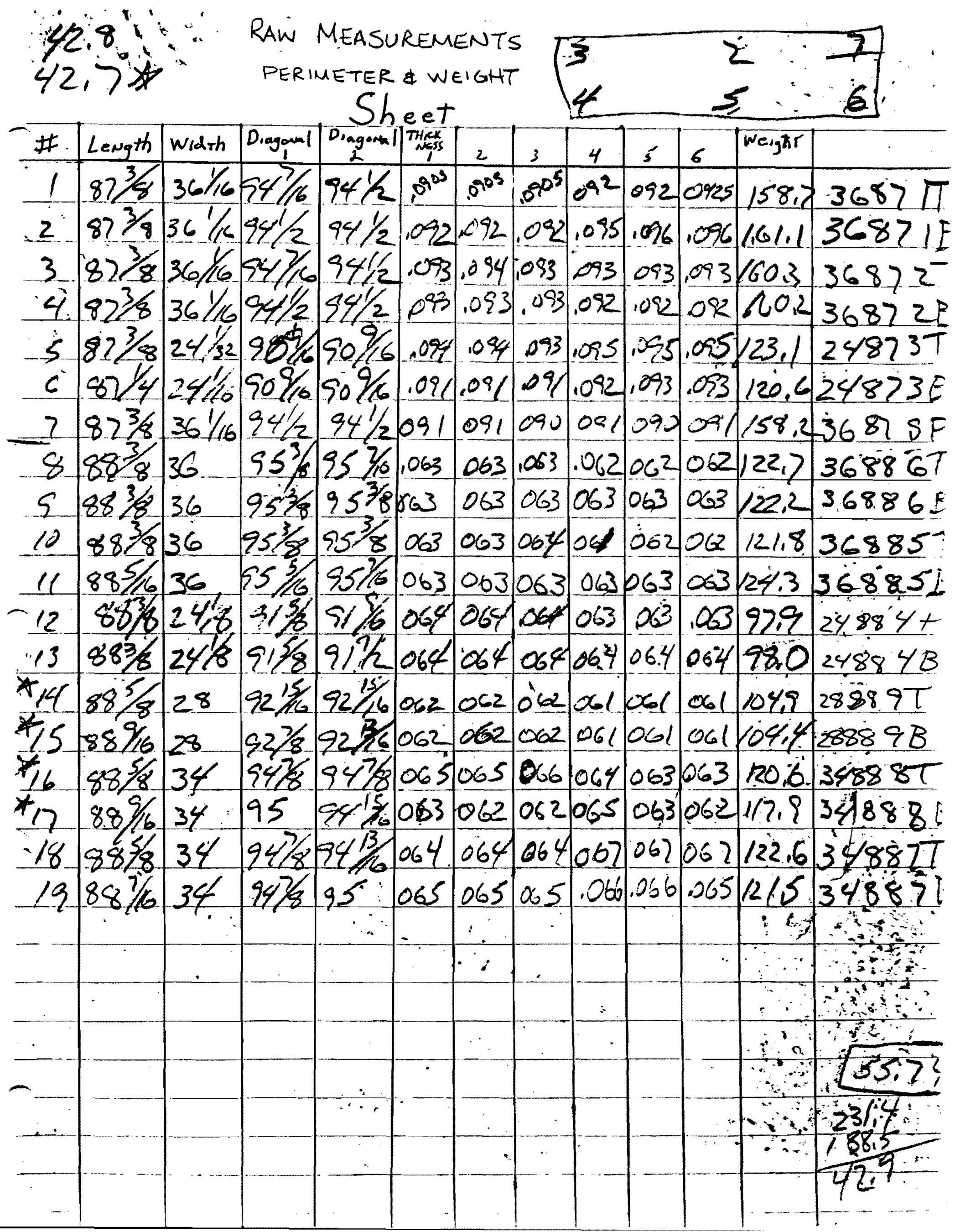




\section{D0 Purchase Requisition}

\section{Requestor Information:}

$\begin{array}{ll}\text { Name: } & \text { Krempetz, Kurt J } \\ \text { ID No.: } & 3679 \\ \text { E-mail: } & \text { krempetz@fnal.gov } \\ \text { Phone: } & 4657\end{array}$

Vendor Information:

$$
\begin{aligned}
& \text { Sol. } O D=1415^{+3} \mathrm{~mm}=55.70866 \mathrm{w} \phi \\
& \text { MEAN RAD } 1=27.85433+3 / 64=27.90121 \text { " } \\
& \pi R=87.654^{\prime \prime}
\end{aligned}
$$

Name: Vulcan Lead Prod Co

Phone: 414-645-2040

Fax:

Street 1:

1400 W Pierce St

Street 2:

Attn:

City State, Zip

Milwaukee WI 53204

Buyers Note:

MEAN RAD $2=27.85433+\frac{3 / 3}{32}+\frac{1}{32}=27.97933$ $\pi R=87.89966^{\prime \prime}$

MEAN ROD $3=27.85+33+3 / 32+1 / 10+1 / 32$ $=28.04183$ $\pi R=88.096^{n}$

\section{Requisition Information:}

Requestor ID:

Entry Date:

ProCard Number:

PO Number:

PO Date:

Requistion Number:

Requistion Date:

Delivery Date:

Date Received:

Last Modified:

Previous PO:

Project Description :

Short Description:

Buyer :

Status :

Urgency :
D0986365

10/27/1997

505339

$11 / 07 / 1997$

106757

$11 / 06 / 1997$

$12 / 03 / 1997$

$02 / 04 / 1998$

Do Upgrade Central Preshower

PRESHOWER LEAD

Johnson

PO

Rush
Help

Desired Delivery Date: 11/17/1997

Line Items:

\begin{tabular}{|l|c|c|r|r|r|r|r|}
\hline Qty & Type & Description & Unit Price & Total & WBS & $\begin{array}{c}\text { \% of } \\
\text { Cost } \\
\text { Est. }\end{array}$ & $\begin{array}{c}\text { Budget } \\
\text { Code }\end{array}$ \\
\hline 2 & Good & $\begin{array}{l}\text { Lead Sheet, .09375" } \\
\text { 3/32"X24"X873/16" }\end{array}$ & 85.25 & 170.501 .1 .3 .9 .2 & 11.67 & DJY \\
\hline
\end{tabular}


‘Do Purchase Requistion

Page 2 of 2

\begin{tabular}{|c|c|c|c|c|c|c|c|c|}
\hline 5 & Good & $\begin{array}{l}\text { Leau sicel, .09375" } \\
\text { B/32"X36"X87 3/16" }\end{array}$ & 85.25 & 426.251 & 1.1.3.9.2 & |11.67 & DJY & \\
\hline 2 & Good & 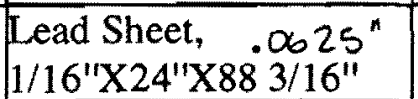 & 85.25 & $170.5 \alpha_{1}$ & 1.1 .3 .9 .2 & 11.67 & DJY & \\
\hline 4 & Good & \begin{tabular}{|l} 
Lead Sheet, \\
$1 / 16 \times 36^{\prime \prime} \times 88$ \\
$3 / 16^{\prime \prime}$
\end{tabular} & 85.25 & 341.001 & 1.1 .3 .9 .2 & 11.67 & DJY & \\
\hline 2 & Good & \begin{tabular}{|l} 
Lead Sheet, $0625^{\prime \prime}$ \\
$1 / 16^{\prime \prime} \mathrm{X} 28^{\prime \prime} \mathrm{X} 88^{\prime}$ \\
$3 / 8^{\prime \prime}$
\end{tabular} & 85.25 & $170.5 q_{1}$ & 1.1.3.9.2 & 11.66 & DJY & \\
\hline 5 & Good & \begin{tabular}{|l} 
Lead Sheet, $.0625^{\prime \prime}$ \\
$1 / 16^{\prime \prime} \times 34^{\prime \prime} 8883 / 8 "$
\end{tabular} & 85.25 & 426.251 & 1.1.3.9.2 & 11.66 & DJY & \\
\hline 0 & Good & $\begin{array}{l}\text { Cut Lead Sheet, } 99.9 \% \\
\text { Pure Lead, Thickness } \\
\text { Tol. +-.003, Width } \\
.0625 \text {, length +-.125 }\end{array}$ & $0.0 \mathrm{~d}$ & 0.00 & & 0.00 & & $\begin{array}{l} \pm .003 \\
\text { Assur } 3 \%\end{array}$ \\
\hline 0 & Good & & 0.00 & 0.00 & & 0.00 & & \\
\hline & Good & & 0.00 & 0.00 & & 0.00 & & \\
\hline \multicolumn{2}{|c|}{$\begin{array}{l}\text { Requisition } \\
\text { Total: }\end{array}$} & & $1,705.00$ & & & & & \\
\hline
\end{tabular}

\section{Comments:}

$03+1$

Contact Sonya Wright for Purchasing questions (sonya@fual.goy, (630) 840-4591)

Contact Jerry OConnell about the operation of this form. (jpoconnall@fnal.gov, (630) 840-3704)

Last Updated: 26-Aug-97

\section{Queries}

GETLINEITEMS (Records $=9$, Time $=31 \mathrm{~ms}$ )

SQL $=$

select LINE_NUM, QUANTITY, PO_LINE_CATAGORY,DESCRIPTION, PRICE,WBS_CODE,BUDGET_CODE, PE from po.req_line_items

WHERE REQUESTOR_ID $={ }^{\prime}$ D0986365'
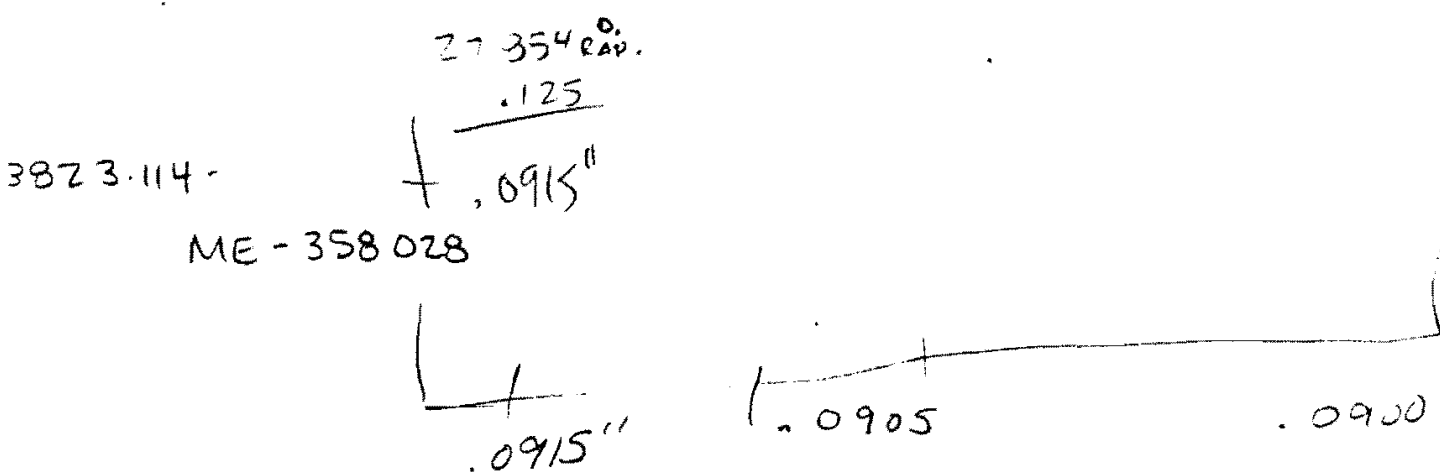

.0905 


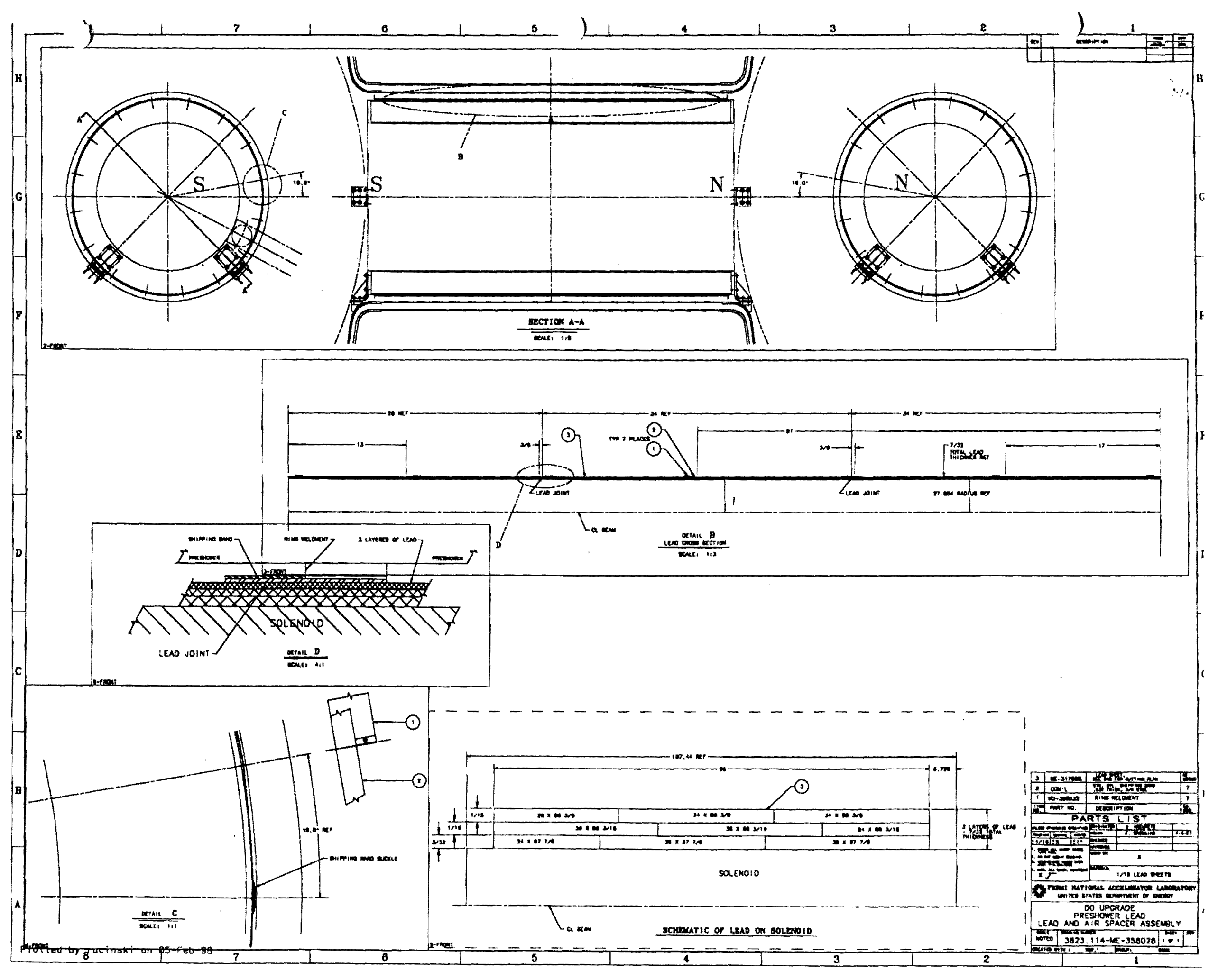


. . ERror Analysis

R.RucinskI 2/6/98

Pres SHOWER LEAS THICKNESS CALC.

$$
t=\frac{P}{\rho W \cdot L}
$$

where

$$
\begin{aligned}
& P=\text { LEAD WT. } \\
& W=\text { LEAS WITH }
\end{aligned}
$$

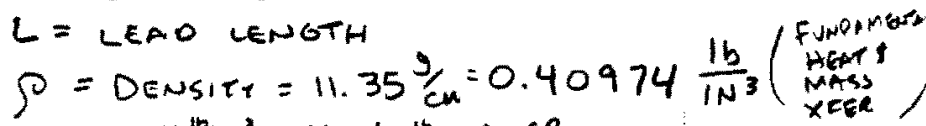

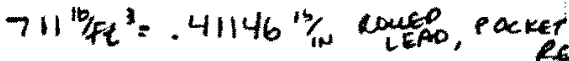

ERror ANALHSIS

REF- EUGINECRENG MEASURE GENTS

$$
S_{E}=\frac{P}{\rho W L} \sqrt{\frac{\left(S_{P}\right)^{2}}{P^{2}}+\left[\frac{S_{\rho W L}}{\rho W L}\right]^{2}}
$$$$
\text { p. } 543 \text { Person }
$$

$$
S_{\rho W L}=w \cdot L \rho \cdot \sqrt{\left[\frac{S_{W}}{W}\right]^{2}+\left[\frac{S_{L}}{L}\right]^{2}+\left[\frac{S_{p}}{\rho}\right]^{2}}
$$

S- error associated with the subscripteo variable

GIVEN:

$$
\begin{aligned}
& S_{w}=S_{L}=1 / 16 "=.0625 \mathrm{w} . \\
& S_{p}=0.51 \mathrm{bs}
\end{aligned}
$$

EXAMPLE WITH FICTITIOUS DATA;

SAM PC LA; $W=36^{\prime \prime} L=87 \frac{3}{16} " \omega T=120.6 \mathrm{kbs}$.

$$
\begin{aligned}
& S_{\rho w L}=(36)(87.1875)(.40974) \sqrt{\left(\frac{.0625}{36}\right)^{2}+\left(\frac{.0625}{87.1875}\right)^{2}+\left[\frac{.002^{16,13^{3}}}{.40974}\right]^{2}} \\
& S_{\rho \omega L}=6.726 \cdot \frac{L B}{1 N} \\
& S_{E}=\frac{120.6 \mathrm{lbs}}{(.40974)(36)(87.1875)} \sqrt{\left[\frac{(0.5)}{120.6}\right]^{2}+\left[\frac{-6.72616 /, N}{(36)(87.1875)(.40974)}\right]^{2}} \\
& S_{E}=.0006 \mathrm{iN}
\end{aligned}
$$

Conclusion: by the proposed meth Hod, the average LEAD THICKNESS CAN bE calculate o TO \pm 1 MIL 